\title{
ANÁLISE DE CRESCIMENTO, INCIDÊNCIA DE Rhizoctonia SP. E EFEITO ANTIXENOSE PARA A FORMIGA-CORTADEIRA Atta sexdens rubropilosa F. EM CLONES DE Eucalyptus grandis $x$ Eucalyptus urophylla TRATADOS COM GIBERELINA ${ }^{1}$
}

Fabio Santos Matos ${ }^{2}$, Débora Zacarias da Silva ${ }^{3}$, Brenda Rodrigues de Souza ${ }^{3}$, Dahís Ramalho Moura ${ }^{3}$, Vinícius de Araújo Lopes ${ }^{3}$, Daniel Diego Costa Carvalho ${ }^{4}$ e Marcio da Silva Araujo ${ }^{4}$

\begin{abstract}
RESUMO - A competitividade do setor florestal brasileiro, fruto das condições climáticas e tecnologia empregada, faz que o país ocupe posição de destaque no cenário mundial. Apesar do elevado crescimento brasileiro no setor florestal, é necessário o desenvolvimento de pesquisas que proporcionem aumento de produtividade. Este estudo objetivou avaliar o efeito fisiológico da aplicação de diferentes concentrações de giberelina $\left(\mathrm{GA}_{3}\right)$ no acúmulo de biomassa do híbrido de Eucalyptus grandis x Eucalyptus urophylla "E. urograndis GG 100" e, também, verificar o efeito da aplicação de hormônio sobre a incidência de Rhizoctonia sp. e sobre efeito antixenose (não preferência) ao corte de folhas pela formiga-cortadeira Atta sexdens rubropilosa. O experimento foi conduzido em bancada a pleno sol, seguindo o delineamento inteiramente casualizado com cinco tratamentos e seis repetições. Mudas clonadas de E. urograndis GG 100, com 120 dias de idade, cultivadas sob bancada a pleno sol em vasos de $12 \mathrm{~L}$, com substrato à base de subsolo, areia e esterco foram tratados com $50 \mathrm{~mL}$ de $\mathrm{GA}_{3}$, nas seguintes concentrações: 0; 50; 100; 150; e $200 \mathrm{mg} \mathrm{L}^{-1}$. Aos 40 dias após a imposição dos tratamentos, as análises foram realizadas. A aplicação de giberelina intensificou o crescimento vegetativo das plantas de eucalipto e promoveu o maior acúmulo de biomassa no mesmo período de tempo de plantas não tratadas. As mudas tratadas com giberelina apresentaram vigoroso crescimento vegetativo, principalmente na concentração de $150 \mathrm{mg} \mathrm{L}^{-1}$. Adicionalmente, as mesmas plantas exibiram maior preferência por formigas-cortadeiras e menor área foliar lesionada pelo fungo Rhizoctonia sp.
\end{abstract}

Palavras-chave: Precocidade; Alongamento; Eucalyptus urograndis.

\section{ANALYSIS OF Rhizoctonia SP. GROWTH, INCIDENCE AND ANTIXENOSIS EFFECT FOR LEAF-CUTTING ANT Atta sexdens rubropilosa F. IN CLONES OF Eucalyptus grandis $x$ Eucalyptus urophylla TREATED WITH GIBBERELLIN}

\begin{abstract}
The competitiveness of the forest sector in Brazil, as a result of climate conditions and technology used, makes the country occupy a prominent position on the world. Despite the high Brazilian growth in the forest sector, the development of research that provide increased productivity is necessary. This study aimed to evaluate the physiological effect of the application of gibberellic acid $\left(G A_{3}\right)$ in the accumulation
\end{abstract}

\footnotetext{
${ }^{1}$ Recebido em 06.07.2013 aceito para publicação em 07.07.2015.

${ }^{2}$ Universidade Estadual de Goiás, Laboratório de Fisiologia Vegetal, Câmpus Ipameri, Ipameri, GO - Brasil. E-mail: <fabio.agronomia@hotmail.com>.

${ }^{3}$ Universidade Estadual de Goiás, Graduação em Engenharia Florestal, Câmpus Ipameri, Ipameri, GO - Brasil. E-mail: $<$ debora_zak@hotmail.com>, <brenda_florestal@hotmail.com>, < dahis_ramalho@hotmail.com $>$ e $<$ viniciusaraujolopes@msn.com> ${ }^{4}$ Universidade Estadual de Goiás, Laboratório de Fitopatologia, Câmpus Ipameri, Ipameri, GO-Brasil.. E-mail: < daniel.carvalho@ueg.br > e $<$ marcio.araujo@ueg.br>.
} 
of biomass of Eucalyptus grandis $x$ Eucalyptus urophylla "E. urograndis GG 100" hybrid and also to verify the effect of this hormone application on the incidence of Rhyzoctonia sp. besides the antixenosis effect (not preferred) on the leaves by leaf-cutting ant Atta sexdens rubropilosa. The experiment was conducted in a bench, full sun, following a completely randomized design with five treatments and six replications. Cloned E. urograndis $G G 100$ seedlings, 120 days old, grown under full sun bench in 12 liter pots with a substrate based on subsoil, sand and manure were treated with $50 \mathrm{ml}$ of $\mathrm{GA} A_{3}$ according to following concentrations: $0,50,100,150$ and $200 \mathrm{mg} \mathrm{L}^{-1}$. Analyzes were performed 40 days after treatment imposition. The application of gibberellin intensified vegetative growth of Eucalyptus plants and promoted a greater accumulation of biomass than untreated plots in the same period of time. Seedlings treated with gibberellin showed vigorous vegetative growth, especially in the concentration of $150 \mathrm{mg} \mathrm{L} \mathrm{L}^{-1}$. In addition, the same plants exhibited higher preference to leaf-cutting ants and smaller leaf area damaged by fungus Rhizoctonia sp.

Keywords: Precocity; Elongation; Eucalyptus urograndis.

\section{INTRODUÇÃO}

A exploração comercial de florestas plantadas dos gêneros Pinus e Eucalyptus apresenta significativa importância para a economia brasileira. As espécies do gênero Eucalyptus destacam-se por apresentar múltiplos usos e potencial para atender aos diversos segmentos da indústria brasileira. A constante elevação do valor econômico e a escassez de madeiras nobres intensificaram o uso diversificado do gênero Eucalyptus (SOUZA et al., 2012). O eucalipto encontrou, no Brasil, as condições ideais para o desenvolvimento. A produtividade dos plantios em terras brasileiras é superior à de países tradicionais, como a Austrália (centro de origem da espécie). A competitividade do setor florestal brasileiro, fruto das condições climáticas e da tecnologia desenvolvida pelas empresas e instituições de pesquisa, faz que o país ocupe posição de destaque no cenário mundial (FERREIRA et al., 2012). O setor florestal responde por $3,5 \%$ do Produto Interno Bruto (PIB) e gera 4,4 milhões de empregos diretos e indiretos (ABRAF, 2012).

Apesar do elevado potencial brasileiro no setor florestal, o país pode produzir mais e transferir riqueza para outros segmentos da economia. Para ser competitivo, conduzindo esse processo de forma consistente e sustentável, faz-se necessário o desenvolvimento de pesquisas que proporcionem aumento de produtividade, abrindo novos mercados, aumentando as exportações e, simultaneamente, modernizando e assegurando elevado padrão social e ambiental às atividades florestais (FERREIRA et al., 2012). A redução da idade de corte e o aumento do acúmulo de biomassa das florestas de eucalipto contribuirão para alavancar o setor e aumentar a participação brasileira no cenário mundial. A obtenção de árvores aptas à colheita em curto espaço de tempo certamente dependerá de alterações fisiológicas da espécie. A utilização de reguladores de crescimento visando melhorar a qualidade da madeira, induzir a floração e retardar ou acelerar o crescimento vegetativo tem sido uma constante em pesquisas científicas de várias espécies (ABDELGADIR etal., 2009; XIONG et al., 2009; GHOSH et al., 2010; KIBA et al., 2010; DOORN et al., 2011; PAN et al., 2011; PEREIRA et al., 2011).

O uso de reguladores vegetais na agricultura tem mostrado grande potencial no aumento da produtividade, embora sua utilização ainda não seja prática rotineira em culturas que não atingiram alto nível tecnológico. Há grande interesse em elucidar a interferência dos reguladores vegetais na lignificação, densidade da madeira, precocidade e rendimento (BIEMELT et al., 2004; PEREIRA et al., 2011).

A elucidação científica dos aspectos físiológicos que norteiam o crescimento da espécie contribuirá para obtenção de material precoce. Pouco se conhece sobre os aspectos morfofisiológicos dos reguladores de crescimento em eucalipto e tampouco sobre o efeito da aplicação desses reguladores sobre agentes limitantes da cultura como pragas e doenças.

Para o eucalipto, sabe-se que as formigas-cortadeiras são capazes de selecionar para corte de folhas, espécies e, até mesmo, procedência de determinadas espécies (SANTANA, 1998). Isso, segundo Hubbell e Wiemer (1983), porque elas são capazes de distinguir sutis diferenças qualitativas e quantitativas nos elementos constituintes das folhas. Em relação ao fungo fitopatogênico Rhizoctonia, agente causal da queima das folhas do eucalipto, sabe-se que ocorre em todas as fases de propagação clonal dessa planta (ALFENAS et al., 2004) e, muitas das vezes, para seu difícil controle, é necessário o uso intensivo de fungicidas. 
Visando reduzir o tempo de corte, acelerar o acúmulo de biomassa com aplicação de concentrações economicamente viáveis, este trabalho objetivou avaliar o efeito fisiológico da aplicação exógena de diferentes concentrações de giberelina $\left(\mathrm{GA}_{3}\right)$ no acúmulo de biomassa de E. grandis x E. urophylla, em sua reação ao patógeno Rhizoctonia sp. e à sua não preferência ao corte pela formiga-cortadeira $A$. sexdens rubropilosa.

\section{MATERIAL E MÉTODOS}

\subsection{Delineamento experimental}

O trabalho foi realizado na Universidade Estadual de Goiás, unidade de Ipameri (Lat. 17 43' 19' S, Long. $48^{\circ} 09^{\prime} 35^{\prime}$ 'W, Alt. $773 \mathrm{~m}$ ), Ipameri, Goiás. Essa região possui clima tropical com inverno seco e verão úmido (Aw), de acordo com a classificação de Köppen. Os clones de "GG 100" oriundos de estacas foram inicialmente conduzidos em tubetes de $50 \mathrm{~cm}^{3}$, com substrato comercial Platmax ${ }^{\circledR}$ ( (substrato à base de casca de Pinus e vermiculita). Após 100 dias, as plantas foram transferidas para vasos de $12 \mathrm{~L}$, contendo como substrato subsolo, areia e esterco, na proporção de $3: 1: 0,5$, respectivamente. O experimento foi realizado em bancada a pleno sol, seguindo o delineamento inteiramente casualizado com cinco tratamentos (plantas de "GG 100" com 120 dias de idade foram tratadas com $50 \mathrm{ml}$ de giberelina, nas concentrações de $0 ; 50 ; 100 ; 150$; e $200 \mathrm{mg} \mathrm{L}^{-1}$ ) e seis repetições. As mudas foram irrigadas diariamente com volume de água correspondente à evapotranspiração diária. O volume de água fornecido à planta foi estimado seguindo recomendações de Allen et al. (2006). Aos 40 dias após a imposição dos tratamentos, as seguintes análises foram realizadas: biomassa total, número de folhas, razões de massa foliar (RMF), caulinar (RMC) e radicular (RMR), diâmetro do caule, altura de planta, reação à Rhizoctonia e teste de preferência por formigacortadeira $A$. sexdens rubropilosa.

\subsection{Variáveis de crescimento}

O número de folhas, comprimento e diâmetro do caule foram mensurados entre 8 e $10 \mathrm{~h}$ da manhã, utilizando régua graduada e paquímetro digital. Folhas, raízes e caules foram destacados e secos em estufa a $72{ }^{\circ} \mathrm{C}$ até atingir massa seca constante e, em seguida, pesados separadamente. Com os dados de massa seca, calculou-se a razão de massa foliar (RMF), razão de massa da raiz ( $R M R$ ), razão de massa do caule (RMC), razão parte aérea/sistema radicular (PA/SR) e biomassa total.

\subsection{Reação das plantas a Rhizoctonia}

Para este teste, foram coletadas, aleatoriamente, cinco folhas do terço médio das plantas de cada tratamento. Em seguida, as folhas foram lavadas em água corrente e deixadas para secar em câmara de fluxo laminar por $10 \mathrm{~min}$ (CARVALHO et al., 2008). Para as inoculações, foram realizados cinco furos no centro do limbo foliar com o auxílio de uma agulha esterilizada e depositado um disco de ágar $(7 \mathrm{~mm})$ contendo micélio de Rhizoctonia (isolado E-65-06), onde permaneceu cinco dias para crescimento. As folhas inoculadas com o patógeno (cinco folhas para cada tratamento) foram submetidas a condições controladas de câmara úmida em caixas acrílicas transparentes (gerbox $-11 \times 11 \mathrm{~cm}$ ), contendo uma folha de papel de germinação. As avaliações da severidade da doença foram realizadas aos quatro dias após a inoculação (DAI), aplicando-se a escala de notas a seguir: $0=$ ausência de lesão nas folhas inoculadas; 1 = lesão na face abaxial da folha com até $25 \%$ da área do disco de ágar inoculado (adai); 2 = lesão na face abaxial da folha com 25 até $50 \%$ da adai; 3 = lesão na face abaxial da folha com 50 até $75 \%$ da adai; 4 = lesão na face abaxial da folha com 75 até $100 \%$ da adai; nota $5=$ a lesão na face abaxial da folha é maior do que na área total do disco de ágar inoculado. Para as análises estatísticas, foi considerado o ponto médio de cada nota atribuída, o que permitiu expressar os resultados em $\mathrm{mm}^{2}$ de área foliar lesionada pelo patógeno.

\subsection{Teste de preferência de formigas-cortadeiras}

A colônia de formiga-cortadeira $A$. sexdens rubropilosa usadas nos testes de não preferência ao corte de folhas foi escolhida de uma criação mantida em fotofase de $12 \mathrm{~h}$, em laboratório da UEG, UnU Ipameri. Essa colônia tinha em torno de 2 anos idade, volume de fungo de cerca de 2 Le, visualmente, com "boa" atividade de forrageamento.

Imediatamente depois de retiradas do terço médio da planta tratada com as diferentes concentrações de giberelina, as folhas eram oferecidas às operárias da formiga-cortadeira em sua arena de forrageamento. Foram selecionadas folhas de tamanho mais uniforme possível. Eram registrados os locais de oferecimento das folhas nas arenas, as quais eram presas nestas com grampos. A posição para oferecimento das folhas (tratamentos) era alterada aleatoriamente. A avaliação da não preferência foi realizada com e sem chances de escolha, ou seja,

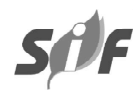

Revista Árvore, Viçosa-MG, v.39, n.5, p.915-922, 2015 
no primeiro caso cinco folhas (uma de cada tratamento) eram oferecidas de uma única vez na arena de forrageamento e, no segundo caso, cinco folhas de um mesmo tratamento eram oferecidas em uma única vez. Essas folhas permaneciam na arena até que uma delas fosse completamente cortada pelas operárias de A. sexdens rubropilosa ou, por um tempo máximo de $30 \mathrm{~min}$, tempo esse definido em teste preliminar para a referida colônia, onde as operárias cortavam por completo uma folha do híbrido de E. grandis $\mathrm{x}$ E. urophylla. Passado esse tempo, as folhas eram retiradas da arena e pesadas.

\subsection{Procedimentos estatísticos}

O experimento foi montado seguindo o delineamento inteiramente casualizado com cinco tratamentos e seis repetições. Os dados foram submetidos à análise de variância e, adicionalmente, procedeu-se à análise de regressão das variáveis com diferença estatística. Todas as análises foram realizadas utilizando o software SISVAR 5.3 (FERREIRA, 2011).

\section{RESULTADOS}

Os resultados da análise de variância de todas as variáveis analisadas encontram-se na Tabela 1 . Independentemente da concentração de giberelina, o número de folhas e razões de massa radicular e foliar não apresentaram diferença estatística entre os tratamentos. A altura da planta, diâmetro do caule, biomassa total, razão de massa caulinar, teste de preferência de formigas-cortadeiras e área foliar lesionada por Rhizoctonia apresentaram diferenças estatísticas em função dos tratamentos utilizados.

Os gráficos com a análise de regressão das variáveis fisiológicas com significativa diferença estatística são mostrados na Figura 1. A altura da planta, o diâmetro do caule e a biomassa total foram superiores a $36 \%$ e $29 \%$ com respeito ao tratamento $150 \mathrm{mg} \mathrm{L}^{-1}$ de giberelina em relação à testemunha. A razão de massa caulinar foi, em média, $36 \%$ superior nas plantas do tratamento que proporcionou maior razão de massa caulinar (200 $\mathrm{mg} \mathrm{L}^{-1}$ de giberelina) em relação à testemunha sem aplicação de giberelina.

O gráfico com a análise de regressão da área foliar lesionada por Rhizoctonia e o teste de preferência para formigas-cortadeiras sem e com opção de escolha são mostrados na Figura 2. A área foliar lesionada por
Rhizoctonia foi, em média, $24 \%$ maior nas plantas do tratamento que proporcionou maior lesão $\left(100 \mathrm{mg} \mathrm{L}^{-1} \mathrm{de}\right.$ giberelina) em relação à testemunha sem aplicação de giberelina. O teste de preferência para formigascortadeiras sem e com opção de escolha foi, em média, $1.207 \%$ e $719 \%$, respectivamente, superior nas plantas do tratamento que proporcionou maior lesão (100 mg $\mathrm{L}^{-1}$ de giberelina) em relação à testemunha sem aplicação de giberelina.

\section{DISCUSSÃO}

A análise fisiológica das plantas no estágio inicial auxilia a identificação de materiais promissores com elevado potencial de produção e contribui para a escolha da espécie adequada nas condições vigentes (PEIXOTO et al., 2006). Nesse contexto, as aplicações de giberelina promoveram alterações significativas no crescimento vegetativo das plantas de eucalipto em todas as doses aplicadas. As plantas tratadas apresentaram os maiores valores de altura da planta, diâmetro do caule e biomassa total. A biomassa da planta é importante variável de crescimento por refletir a assimilação de carbono ao longo do tempo (PEIXOTO et al., 2006; MATOS et al., 2011). Essas variáveis em conjunto tornaram o crescimento vegetativo das plantas tratadas mais vigoroso. A aplicação de giberelina altera uma série de caracteres produtivos, como o crescimento vegetativo, a densidade da madeira, o teor de lignina e o diâmetro da fibra de plantas de Eucalyptus (ALONI, 1979; BIEMELT et al., 2004; SANTOS, 2005; PEREIRA et al., 2011), no entanto o intenso alongamento do caule elevando a altura das plantas é o principal efeito da aplicação de giberelina (HIGASHI et al., 2002; OLIVEIRA et al., 2005).

A maior partição de assimilados e, consequentemente, maior biomassa para o crescimento e desenvolvimento do caule e em menor proporção do sistema radicular constituiu marcante alteração oriunda da aplicação de giberelina. O desenvolvimento de um sistema radicular vigoroso é característica indispensável para adaptação das mudas às condições de campo (FREITAS et al., 2009), no entanto o intenso desenvolvimento da parte aérea indica que a menor partição de biomassa para o sistema radicular não limitou a absorção de água e nutrientes. A menor partição de assimilados para o sistema radicular resultou em maior disponibilidade desses para crescimento e desenvolvimento do caule em altura e diâmetro. $\mathrm{O}$ elevado acúmulo de biomassa associado ao expressivo 
Tabela 1 - Resumo da análise de variância da altura de planta, diâmetro do caule, número de folhas, biomassa total, razão de massa radicular (RMR), razão de massa caulinar (RMC), razão de massa foliar (RMF), teste de preferência de formigas-cortaderiras por folhas sem opção de escolha (TPSOC) e teste de preferência p formigas-cortadeiras com opção de escolha (TPCOC) e área de lesão foliar causada por Rhizoctonia (ALF) e de plantas de eucalipto (GG 100) tratadas com diferentes concentrações de giberelina ${ }^{(1)}$.

Table 1 - Analysis of variance for plant height, stem diameter, number of leaves, total biomass, root mass ratio (RMR), stem mass ratio $(R M C)$ and leaf weight ratio $(R M F)$, preference test of leaf-cutting ants for leaves without option to choose (TPSOC), preference test of leaf-cutting ants with option to choose (TPCOC) and leaf lesion area caused by Rhizoctonia (ALF) and eucalyptus plants (GG 100) treated with different concentrations of gibberellin ${ }^{(I)}$.

\begin{tabular}{|c|c|c|c|c|c|c|c|c|c|c|c|}
\hline \multirow{2}{*}{ Concentrações $\left(\mathrm{mg} \mathrm{L}^{-1}\right)$} & \multicolumn{11}{|c|}{ Quadrados médios } \\
\hline & & Altura $(\mathrm{cm})$ & Diâmetro(mm) & $\mathrm{N}^{\circ}$ de folhas & Biomassa $(\mathrm{g})$ & RMR & $\mathrm{RMC}$ & $\mathrm{RMF}$ & TPSOC & TPCOC & ALF \\
\hline Concentrações & 4 & $932,8^{*}$ & $11,8^{*}$ & $8100 \mathrm{~ns}$ & $1518^{*}$ & $0,01 \mathrm{~ns}$ & $0,01 *$ & $0,002 \mathrm{~ns}$ & 19691* & $23646 * *$ & $364,07 * *$ \\
\hline Resíduo & 25 & 135,7 & 2,2 & 5754 & 587 & 0,006 & 0,003 & 0,002 & 2990 & 1984 & 36,9 \\
\hline $\mathrm{CV}(\%)$ & & 10,4 & 11,1 & 23,6 & 23,8 & 25,6 & 12,8 & 16,2 & 33,0 & 36,0 & 26,8 \\
\hline 0 & & $94,7(100)$ & $11,9(100)$ & $320,5(100)$ & $96,3(100)$ & $0,34(100)$ & $0,33(100)$ & $0,33(100)$ & $13(100)$ & $26(100)$ & $24(100)$ \\
\hline 50 & & $107,3(113)$ & $12,5(105)$ & $332,7(104)$ & $99,1(103)$ & $0,32(94)$ & $0,38(115)$ & $0,30(91)$ & $25(193)$ & $17(71)$ & $25,9(108)$ \\
\hline 100 & & $114,2(121)$ & $14,4(121)$ & $313,3(98)$ & $108,1(112)$ & $0,35(103)$ & $0,37(112)$ & $0,28(85)$ & 157 (1207) & $187(719)$ & $29,8(124)$ \\
\hline 150 & & $128,7(136)$ & $15,3(129)$ & $370,7(116)$ & $124,7(129)$ & $0,27(79)$ & $0,43(130)$ & $0,30(91)$ & $94(723)$ & $88(338)$ & $12,5(52)$ \\
\hline 200 & & $116,2(123)$ & $14,4(121)$ & $268,7(84)$ & $81,0(84)$ & $0,24(71)$ & $0,45(136)$ & $0,31(94)$ & $18(138)$ & $54(207)$ & $10,6(44)$ \\
\hline
\end{tabular}

${ }^{*} \mathrm{e}^{* *}$ Significativos a $5 \%$ e $1 \%$ de probabilidade, respectivamente. Os valores entre parênteses representam percentual sobre o controle. $*$ e $* *$ Significant at $5 \%$ and $1 \%$ probability levels, respectively. Values in parentheses represent percentage of control.
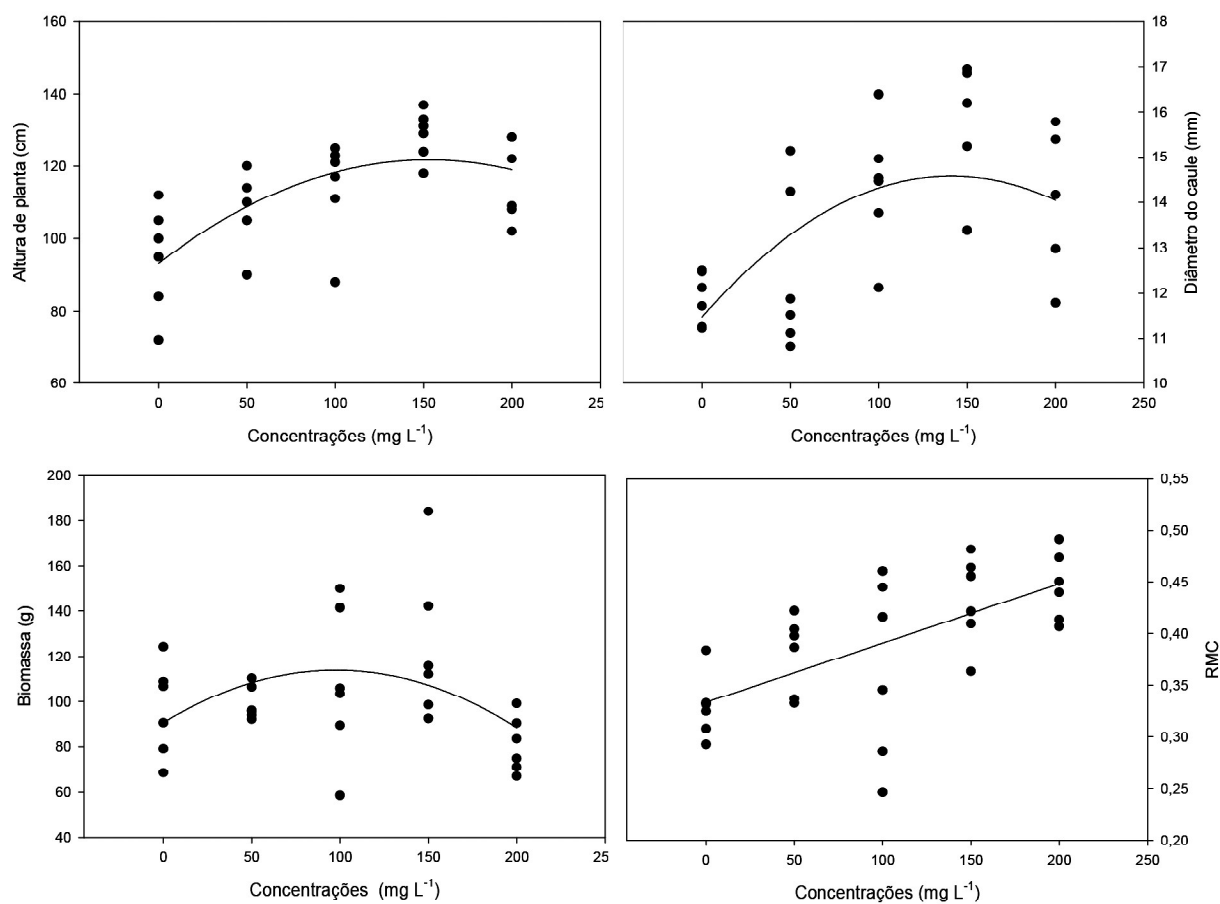

Figura 1 - Equações de regressão para altura de plantas $\left(Y=93,24+0,37 x-0,0012 x^{2}, r^{2}=0,85^{* *}\right)$, diâmetro do caule $(Y=11,48+$ $\left.0,044 \mathrm{x}-0,0002 \mathrm{x}^{2}, \mathrm{r}^{2}=0,95^{*}\right)$ e biomassa total $\left(\mathrm{Y}=90,71+0,47 \mathrm{x}-0,0024 \mathrm{x}^{2}, \mathrm{r}^{2}=0,70^{*}\right)$ e razão de massa caulinar $\left(\mathrm{Y}=0,33+0,0006 \mathrm{x}, \mathrm{r}^{2}=0,72 *\right)$ de eucalipto $(\mathrm{GG} 100)$ tratadas com diferentes concentrações de giberelina. *

$=$ regressão significativa $(0.01<\mathrm{p} \leq 0.05) ; * *=$ regressão significativa $(\mathrm{p} \leq 0.01)$.

Figure 1-Regression equations for plant height $\left(Y=93,24+0,37 x-0,0012 x^{2}, r^{2}=0,85 * *\right)$, stem diameter $(Y=11,48+0,044 x$ $\left.0,0002 x^{2}, r^{2}=0,95^{*}\right)$ and total biomass $\left(Y=90,71+0,47 x-0,0024 x^{2}, r^{2}=0,70^{*}\right)$ and mass ratio stem $(Y=0,33+$ $\left.0,0006 x, r^{2}=0,72 *\right)$ for Eucalyptus urograndis (GG 100) treated with different concentrations of gibberellin. $*=$ significant regression $(0.01<p \leq 0.05) ; * *=$ significant regression $(p \leq 0.01)$. 

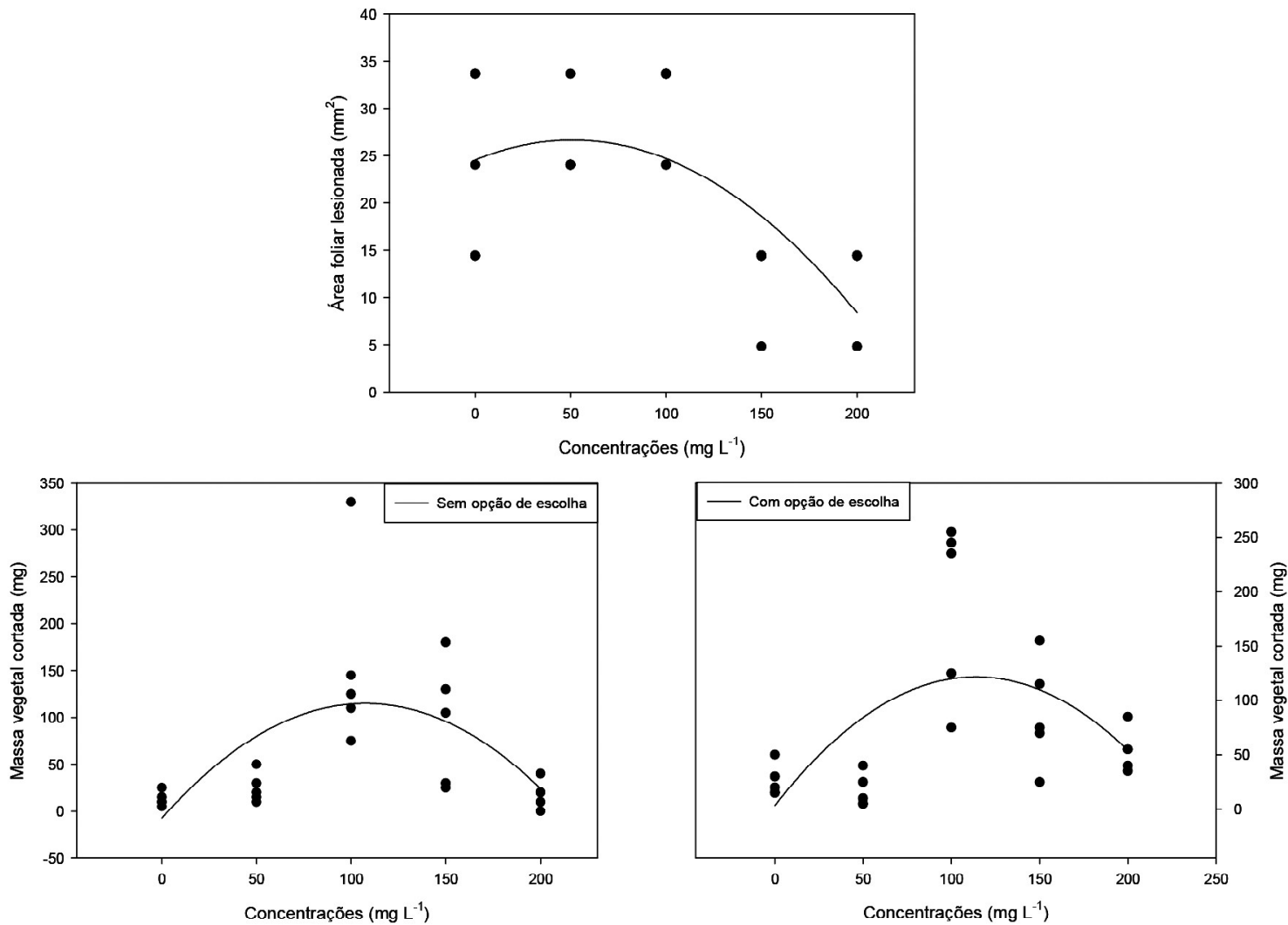

Figura 2 - Equações de regressão da área de lesão foliar causada por Rhizoctonia $\left(\mathrm{Y}=24,53+0,084-0,0008 \mathrm{x}^{2} \mathrm{R}^{2}=0,91 * *\right)$, teste de preferência de formigas-cortadeiras por folhas sem opção de escolha $\left(Y=-7,40+2,278 x-0,0106 x^{2}\right.$, $\left.\mathrm{R}^{2}=0,70^{*}\right)$ e teste de preferência de formigas-cortadeiras com opção de escolha $\left(\mathrm{Y}=3,43+2,08 \mathrm{x}-0,0091 \mathrm{x}^{2}\right.$, $\left.\mathrm{rR}^{2}=0,71^{*}\right)$ de plantas de eucalipto (GG 100) tratadas com diferentes concentrações de giberelina. * = regressão significativa $(0.01<\mathrm{p} \leq 0.05) ; \mathrm{e}^{* *}=$ regressão significativa $(\mathrm{p} \leq 0.01)$.

Figure 2 - Regression equations for Leaflesion area caused by Rhizoctonia $\left(Y=24,53+0,084-0,0008 x^{2} R^{2}=0,91 * *\right)$, Preference test of ants per plant leaves no option to choose $\left(Y=-7,40+2,278 x-0,0106 x^{2}, R^{2}=0,70^{*}\right)$ and Preference test of ants per plant leaves with option to choose $\left(Y=3,43+2,08 x-0,0091 x^{2}, r R^{2}=0,71 *\right)$ Eucalyptus urograndis (GG 100) plants treated with different concentrations of gibberellin. ${ }^{*}=$ significant regression $(0.01$ $<p \leq 0.05) ;$ and $* *=$ significant regression $(p \leq 0.01)$.

crescimento do caule em altura e diâmetro é de grande interesse da Engenharia Florestal pelo efeito direto na precocidade, qualidade e produtividade da madeira de Eucalyptus. A aplicação de giberelina altera o crescimento vegetativo das plantas, intensificando o desenvolvimento da parte aérea (BIEMELT et al., 2004; SPONSEL, 2006).

O teste de preferência de formigas-cortadeiras por folhas de eucalipto e a incidência do fungo Rhizoctonia $\mathrm{sp}$. indicam que as folhas das plantas tratadas tiveram maior preferência (com e sem opção de escolha) em relação à testemunha. A aplicação do regulador alterou o crescimento vegetativo das plantas de eucalipto, tornando-o vigoroso. Possivelmente a alocação de assimilados para o intenso crescimento vegetativo esteja relacionada com a menor produção de algum metabólito (fenóis, alcaloides e esteróis) que altera o metabolismo vegetal, tornando-o preferencial ao ataque de formigascortadeiras. Resultados diferentes foram observados entre tratamentos neste estudo quanto à severidade do fungo Rhizoctonia sp., que avançou com menor intensidade sobre os tecidos foliares de plantas de 
eucalipto de elevada altura, diâmetro do caule e biomassa (plantas tratadas com $150 \mathrm{mg} \mathrm{L}^{-1} \mathrm{de}$ giberelina), indicando que o vigoroso crescimento vegetativo não tornou as plantas suscetíveis ao fungo Rhizoctonia sp.

A aplicação de giberelina intensifica o crescimento vegetativo das plantas de eucalipto e pode tornar o vegetal precoce, por promover maior acúmulo de biomassa em mesmo período de tempo em relação às plantas não tratadas. No entanto, as plantas tratadas apresentaram maior preferência pelas formigas-cortadeiras. Pesquisas com maior refinamento são necessárias para maior elucidação dos metabólicos secundários envolvidos na proteção de plantas de eucalipto contra o ataque de pragas e a viabilidade econômica da aplicação de giberelina.

\section{CONCLUSÕES}

As mudas tratadas com giberelina apresentaram vigoroso crescimento vegetativo, principalmente na concentração de $150 \mathrm{mg} \mathrm{L}^{-1}$. Além disso, elas foram preferidas pelas formigas-cortadeiras e apresentaram baixa área foliar lesionada pelo fungo Rhizoctonia sp.

\section{AGRADECIMENTOS}

À Universidade Estadual de Goiás (UEG), pelo apoio financeiro dispensado para a realização deste trabalho; e à Ceres Florestal, pela doação dos clones utilizados nesta pesquisa.

\section{REFERÊNCIAS}

ABDELGADIR, H.A.; JOHNSON, S.D.; VAN STADEN, J. Effect of foliar application of plant growth regulators on flowering and fruit set in Jatropha curcas - A potential oil seed crop for biodiesel. Plant Growth Regulation, v.58, n.3, p.287-295, 2009.

\section{ASSOCIAÇÃO BRASILEIRA DE PRODUTORES DE FLORESTAS PLANTADAS - ABRAF.}

Disponível em: www.abraflor.org.br. Acesso em: 30 de jun. 2012.

ALLEN, R.G.; PRUIT, W.O.; WRIGHT, J.L.; HOWELL, T.A.; VENTURA, F.; SNYDER, R.; ITENFISU, D.; STEDUTO, P.; BERENGENA, J.;
YRISARRY, J.B.; SMITH, M.; PEREIRA, L.S.; RAES, D.; PERRIER, A.; ALVES, I.; WALTER, I.; ELLIOTT, R.A Recommendation on standardized surface resistance for hourly calculation of reference ETo by the FAO56 Penman-Monteith method. Agricultural Water Management, v. 81, n.1, p.1-22, 2006.

ALFENAS, A.C.; ZAUZA, E.A.V.; MAFIA, R.G.; ASSIS, T.F. Clonagem e doenças do eucalipto. Viçosa, MG: Universidade Federal de Viçosa, 2004.

ALONI, R. Role of auxin and gibberellin in differentiation of primary phloem fibers. Plant Physiology, v.4, n.63, p.609-614, 1979.

BIEMELT, S.; TSCHIERSCH, H.; SONNEWALD, U. Impact of altered gibberellin metabolism on biomass accumulation, lignin biosynthesis, and photosynthesis in transgenic tobacco plants. Plant Physiology, v.135, n.1, p.254-265, 2004.

CARVALHO, D.D.C.; ALVES, E.; BATISTA, T.R.S.; CAMARGOS, R.B.; LOPES, E.A.G.L. Comparison of methodologies for conidia production by Alternaria alternata from citrus. Brazilian Journal of Microbiology, v.39, n.4, p.792-798, 2008.

DOORN, W.G.V.; PERIK, R.R.J.; ABADIE, P.; HARKEMA, H. A. Treatment to improve the vase life of cut tulips: Effects on tepal senescence, tepal abscission, leaf yellowing and stem elongation. Postharvest Biology and Technology, v. 61, n.1, p.56-63, 2011.

FERREIRA, D.F. Sisvar: a computer statistical analysis system. Ciência e Agrotecnologia, v.35, p.1039-1042, 2011.

FERREIRA, S.M.; PETRAUSKI, C.; MARQUES, G.M.; SILVA, M.L.; CORDEIRO, S.A.; SOARES, N.S. Competitividade do Brasil no mercado internacional de madeira serrada. Cerne, v. 18 , n.1, p.99-104, 2012.

FREITAS, T.A.S.; BARROSO, D.G.; CARNEIRO, J.G.A.; PENCHEL FILHO, R.M.; SOUZA, L.S. Efeito de deformação e poda das raízes de mudas de eucalipto sobre o crescimento no campo. Floresta, v.39, n.2, p.355-363, 2009.

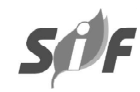

Revista Árvore, Viçosa-MG, v.39, n.5, p.915-922, 2015 
GHOSH, A.; CHIKARA, J.; CHAUDHARY, D.; PRAKASH, A.; BORICHA, G.; ZALA, A. Paclobutrazol arrests vegetative growth and unveils unexpressed yield potential of Jatropha curcas L. Journal of Plant Growth Regulation, v.29, n.3, p.307-315, 2010.

HIGASHI, E.N.; SILVEIRA, R.L.V.A.; GOUVÊA, C.F.; BASSO, L.H.M. Ação fisiológica de hormônios vegetais na condição hídrica, metabolismo e nutrição mineral. In:

Introdução à fisiologia do desenvolvimento vegetal. Maringá: EDUEM, 2002.

HUBBELL, S.P.; WIEMER, D.F. Host plant selection by attine ant. In: JAISSON, P. (Ed.) Social insects in the tropics. Paris: University of Paris Press, 1983. p.134-154.

KIBA, T.; SAKAKIBARA, H. Role of cytokinin in the regulation of plant development. In: PUA, E.C.; DAVEY, M.R. Plant developmental biology-biotechnological perspectives. New York: Springer, 2010. p.237-254.

MATOS, F.S.; GAMBOA, I.; RIBEIRO, R.P.; MAYER, M.L.; NEVES, T.G.; LEONARDO, B.R.L.; SOUZA, A.C. Influência da intensidade luminosa no desenvolvimento de mudas de Jatropha curcas L. Agrarian, v.4, n.14, p.265272, 2011.

OLIVEIRA, A.; FERREIRA, G.; RODRIGUES, J.D.; FERRARI, T.B.; KUNZ, V.L.; PRIMO, M.A.; POLETTI, L.D. Efeito de reguladores vegetais no desenvolvimento de mudas de Passiflora alata Curtis. Revista Brasileira de Fruticultura, v.27, n.1, p.9-13, 2005.

PAN, Z.B.; XU, Z.F. Benzyladenine treatment significantly increases the seed yield of the biofuel plant Jatropha curcas. Journal Plant Growth Regulation, v.30, n.1, p.166-174, 2011.
PEIXOTO, C.P.; CERQUEIRA, E.C.; SOARES FILHO, W.S.; CASTRO NETO, M.T.; LEDO, C.A.S.; MATOS, F.S.; OLIVEIRA, J.G. Análise de crescimento de diferentes genótipos de citros cultivados sob déficit hídrico. Revista Brasileira de Fruticultura, v.28, n.3, p.439-443, 2006.

PEREIRA, R.P.W.; ABREU, H.S.; MONTEIROS, B.O.; SOUZA, N.D. Variação ligno-anatômica em mudas de eucalyptus grandis tratadas com reguladores de crescimento. Cerne, v.17, n.3, p.369-375, 2011 .

SANTANA, D.A.Q. Resistência de Eucalyptus spp. às formigas Atta sexdens rubropilosa Forel, 1908 e Atta laevigata (F. Smith, 1858) (Hymenoptera:

Formicidae). 1988. 95f. Dissertação ((Mestrado em Ciências) - Universidade Federal de Viçosa, Viçosa, MG, 1988.

SANTOS, S.R. Influência da qualidade da madeira de híbridos de Eucalyptus grandis x Eucalyptus urophylla e do processo Kraft de polpação na qualidade da polpa branqueada. 2005. 178f.

Dissertação (Mestrado em Recursos Florestais) - Escola Superior de Agricultura "Luiz de Queiroz”, Piracicaba, 2005.

SOUZA, J.T.; TREVISAN, R.; DENARDI, L.; STANGERLIN, D.M.; VIVIAN, M.A.; HASELEIN, C.R.; SANTINI, J.E. Qualidade da madeira serrada provenientes de árvores dominantes e média de Eucalyptus grandis submetidos a secagem.

Cerne, v. 18, n.1, p.167-174, 2012.

SPONSEL, V. Gibberellins: Regulators of plant height and seed germination. Plant Physiology, v.4, p.509-538, 2006.

XIONG, G.; LI, J.; WANG, Y. Advances in the regulation and crosstalks of phytohormones. Chinese Science Bulletin, v.54, p.27182733, 2009. 